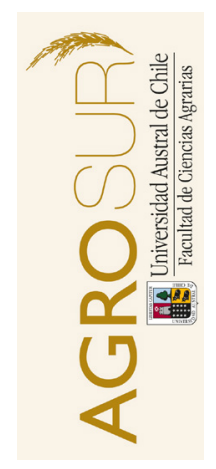

\title{
El liderazgo de las mujeres y los jóvenes rurales de la región Andina de Colombia
}

\author{
The leadership of women and the rural youth of the Andean region of Colombia
}

\author{
Vélez, J. ${ }^{a *}$, Burbano, H. ${ }^{a}$, Navia, J. ${ }^{a}$, Gómez, E. ${ }^{b}$ \\ ${ }^{a}$ Facultad de Ciencias Agrícolas, Universidad de Nariño, Ciudad Universitaria Torobajo, \\ Calle 18 \# 50-02, Pasto, Nariño, Colombia. \\ ${ }^{b}$ Facultad de Ciencias Agropecuarias, Universidad Nacional de Colombia, Sede Palmira, \\ Carrera 32 \# 12-00, Palmira, Colombia.
}

ARTICLE INFO

Article history:

Received 17.09.2018

Accepted 31.10.2018

Keywords:

Gender, leader

Rooting

Displacement

Original Research Article,

Special Issue: Agroecology and

Sustainable Agricultural Systems

*Corresponding author:

Jorge Vélez L.

E-mail address:

jvelezlozano@gmail.com

\section{A B S T R A C T}

The strengthening of local organizations is recognised as one of the key objectives for promoting sustainable rural development. However, in order to achieve this, it is necessary to visualise the leadership of women and young rural people, a fact that is not valued. This research was carried out in the municipality of Alban, Nariño, Colombia, where 67 people were sampled using semistructured surveys. The information was processed using frequency distribution tables and multiple correspondence analyses. The women and young people of the Agricultural Association of Producers of Panela de Alban (APROPAL) showed a vision of empowerment and cultural perspective of the Organization, based on their qualities of production and reproductive life; promoting the conservation desire of nature within the association. There was also a strengthening of the leadership of women and the rural youth of the Organization which is key for improving the associative management in the agro-productive sector, and it constitutes a complementary aspect by strengthening the business sector of the Region. Finally, the participation of women and young people who are part of APROPAL is set up as an important and solid actor in local rural life, opening new ways of social participation and public influence by channeling diverse potentialities and interests.

\section{RESUMEN}

El fortalecimiento de las organizaciones locales es reconocido como uno de los objetivos fundamentales para promover el desarrollo rural sostenible, no obstante, para lograrlo se necesita visibilizar el liderazgo de la mujer y el joven rural, hecho que no se ha valorado. Por ello se realizó esta investigación en el municipio de Albán, Nariño, Colombia, donde se muestrearon 67 personas aplicando encuestas semiestructuradas, procesándose mediante tablas de distribución de frecuencia y análisis de correspondencia múltiple. Se evidenció en las mujeres y los jóvenes de la Asociación Agropecuaria de Productores de Panela de Albán (APROPAL), una visión de empoderamiento y perspectiva cultural de la organización, basada en su calidad de productoras y reproductoras de vida, suscitando en la asociación el deseo conservacionista de la naturaleza; además, se apreció que el fortalecimiento del liderazgo de la mujer y el joven rural de la organización, resulta estratégico para mejorar la gestión asociativa en el sector agroproductivo y constituye un aspecto complementario al fortalecer el tejido empresarial de la región. Finalmente, la participación de las mujeres y jóvenes que hacen parte de APROPAL, se configura como un importante y afianzado actor de la vida local rural y abre nuevas vías de participación social y de influencia pública al canalizar diversas potencialidades e intereses.

Palabras clave: Género, líder, arraigo, desplazamiento.

\section{INTRODUCCIÓN}

La situación de desigualdad social en el campo colombiano, escenario principal del conflicto armado, ha convertido a las mujeres y jóvenes rurales en sujetos para el destierro y el despojo, así como para la desarticulación de las familias, la agricultura familiar y la economía campesina (Benítez, 2010). Ellos se han visto afectados por diversas dinámicas de violencia de los actores armados al propiciar la ruptura de las identidades, desplazamiento forzado, heridas psicológicas y emocionales a largo plazo, que han puesto a prueba la capacidad de resiliencia de este grupo poblacional (Cediel et al., 2017).

De acuerdo con el informe de las Naciones Unidas en el año 2010 se evidencia que, para el año 1997 Colombia, comenzó a registrar a los desplazados internos y que para el año 2010 contaba con un total de $3,3 \mathrm{mi}-$ 
llones (ACNUR, 2010); además, la agencia presidencial para la acción social y la cooperación internacional junto con la consultoría para los derechos humanos y el desplazamiento (CODHES), registró 2.412.834 personas que se encontraban en situación de desplazamiento para el periodo de 2002-2009. En el 2014, el país tiene una de las mayores poblaciones en situación de desplazamiento con seis millones de personas, y para el mes de febrero de 2017 el registro de personas afectadas por estas condiciones fue de 7.108.181, lo que representa un $88,3 \%$ del total de personas reportadas como víctimas del conflicto armado, sobre un total de 8.048.252 (Alpargatero, 2011).

Se considera entonces que los grupos armados junto con las Fuerzas Armadas hacen uso de la fuerza contra la población civil, obligándola a tomar partido y presionándola a dividirse y convertirse en un frente de guerra; además, con los grupos armados se comparten espacios geográficos, recursos económicos, vínculos familiares y culturales. Por tanto, este tipo de violencia genera una ruptura del tejido social, pérdida de cooperación, solidaridad y confianza, generando prevención entre las comunidades, miedo a opinar y debilitamiento en procesos asociativos de la población, lo que conlleva al empobrecimiento y al empeoramiento de las condiciones de vida (González et al., 2016).

Por otra parte, aquellas personas que no consideran la posibilidad de participar en estos grupos armados, resultan obligadas a salir de sus territorios y asentarse en lugares que son completamente ajenos a sus modos de vida, lo que implica un desplazamiento forzoso que es considerado como una manifestación de irrespeto sistemático de los actores armados al Derecho Internacional Humanitario (Acuña, 2013).

Es el caso de las mujeres y jóvenes quienes sufren con más intensidad dichas problemáticas, ya que el desplazamiento les dificulta su fácil adaptación a otras zonas y muchas veces resulta frustrante, mientras que por otra parte y aún más preocupante, la vía tomada a causa del conflicto armado por dicha población es la vinculación a los grupos armados ilegales, cifra registrada en 8.098 personas para el año 2017. Así, la falta de oportunidades y la marginación en esta población no permite el progreso en las zonas rurales, ya que a través del desarrollo de sus capacidades, son ellos quienes pueden llegar a posibilitar que el desarrollo social, político y económico, sobre todo en estas áreas no se frustre más. Por este motivo se considera necesario ampliar la información sobre esta población que se ve como una de las más vulnerables (Güechá et al., 2017).

A pesar de la problemática presentada, en el mundo rural de hoy las relaciones de género se están transformando. Ahora se hace más visible la participación de las mujeres y jóvenes en las actividades productivas y en la toma de decisiones. La presencia de las mujeres y jóvenes en la agricultura y ganadería es mayor y mucho más visible hoy que antes, pero no sólo esto, también hay un surgimiento de lo que en la teoría de la nueva ruralidad se llama la "pluriactividad" en el medio rural; tanto mujeres como hombres incursionan en actividades no agrícolas para generar ingresos y adelantar procesos de liderazgo y autogestión (Farah y Pérez, 2003).

Al respecto, Zuluaga-Sánchez y Arango-Vargas (2013) en su investigación "Mujeres campesinas: resistencia, organización y agroecología en medio del conflicto armado" evaluaron dos procesos realizados con la Asociación de Mujeres Organizadas de Yolombó (AMOY) y encontraron que las mujeres se han posicionado como productoras, pero ello tiende a despolitizarse, dado que su trabajo se percibe como una extensión de su rol de cuidadoras, lo que obstaculiza el logro de mayores transformaciones sociales en cuanto a la consecución de la igualdad de género (Zuluaga-Sánchez y Arango-Vargas, 2013).

El fortalecimiento de las organizaciones locales, es reconocido como uno de los factores fundamentales para promover el desarrollo rural sostenible; sin embargo, para lograrlo se necesita visibilizar el liderazgo de las mujeres y los jóvenes rurales, hecho que no se ha valorado. Por lo tanto, se realizó esta investigación con el objetivo de visibilizar el liderazgo de las mujeres y jóvenes rurales, como gestores en la reconstrucción del tejido social en el campo para combatir la pobreza.

\section{MATERIAL Y MÉTODOS}

\section{Localización}

El municipio de Albán está localizado en el nororiente del departamento de Nariño a $53 \mathrm{~km}$ de la ciudad de San Juan de Pasto. Tiene una extensión de 83 km², ubicado a $1971 \mathrm{msnm}$ y una temperatura promedio de $22^{\circ} \mathrm{C}$. El área de estudio se sitúa en las coordenadas $1^{\circ} 28^{\prime} 00^{\prime \prime} \mathrm{LN}$ y $77^{\circ} 05^{\prime} 45^{\prime \prime} \mathrm{LO}$, la densidad demográfica es de 232,57 habitantes por cada $\mathrm{km}^{2}$, el $45,53 \%$ de los habitantes tienen las necesidades básicas insatisfechas (NBI) (Gómez, 2016).

\section{Población}

La investigación se realizó con los 67 miembros de la Asociación Agropecuaria de Productores de Panela de Albán- APROPAL, microempresa asociativa rural sin ánimo de lucro que se constituyó el 14 de agosto del año 2012.

\section{Identificación de la capacidad de liderazgo de la mujer}

Para identificar la capacidad de liderazgo de la mujer dentro de la asociación, se tuvo en cuenta la información socioeconómica obtenida a partir de la meto- 
dología propuesta por Somarriba (2009) y Montenegro (2012). La estructura de recolección de información estuvo constituida por identificación, estado civil de las mujeres y la conformación de su hogar, educación, condiciones laborales y de vivienda y toma de decisiones dentro del hogar, adicionalmente, se buscó indagar, las percepciones sobre calidad de vida y los instrumentos de liderazgo en su asociación y al interior de sus hogares, a través de estrategias lúdicas en forma de talleres, de acuerdo con la metodología propuesta por Burgos y Guerrero (2007).

Fortalecimiento del emprendimiento juvenil, para la reedificación de sus objetivos y propósitos comunitarios

Para el fortalecimiento del emprendimiento juvenil en los jóvenes Albanitas, se trabajaron dos componentes: el primero relacionado con el análisis de la capacidad de emprendimiento de los jóvenes de la asociación $y$, el segundo, con el desarrollo de un plan de trabajo para identificar y generar ideas de negocio en las que estén interesados, a partir de la oferta de la región (Geilfus, 2002; Rosero, 2015).

Los dos enfoques de investigación utilizados fueron el inductivo y deductivo, puesto que se partió de la observación de situaciones particulares relacionadas con la actualidad de los jóvenes de la asociación, como el aprovechamiento de su tiempo libre, así como la disponibilidad de oportunidades y diversidad de negocio y emprendimiento en su comunidad. Como fuentes primarias se tomaron las opiniones de las personas entre los 15 y 24 años de edad pertenecientes a la asociación $\mathrm{y}$ como fuentes secundarias se analizaron los diferentes documentos que involucraron políticas para el trabajo con jóvenes, como información del Fondo Emprender, Colombia Joven, La Red Colombiana de Jóvenes Rurales, Propaís y el Plan de Desarrollo del Municipio de Albán, que apoyaron los conceptos de emprendimiento e idea de negocio.

\section{Análisis de capacidades de emprendimiento juvenil}

Con este propósito se adaptó un cuestionario de análisis de habilidades de emprendedores, siguiendo la propuesta de Castro y Escobar (2015), abordando los siguientes aspectos: 1) Aprovechamiento de nuevas oportunidades; 2) Persistencia y capacidad para enfrentar problemas; 3) Determinación de metas; y 4) Autoconfianza.

\section{Identificación y generación de ideas de negocio}

Se estableció un "marco" de observación participante, en colaboración con todo el equipo de trabajo, para concentrar sus observaciones, y así comparar entre ellos lo que han aprendido, siguiendo la metodología de Geilfus (2002), donde se plantearon las siguientes preguntas:

¿Qué se quiere aprender? ¿En qué actividad y con cuáles personas se debe integrar para buscar respuestas? ¿Cuáles son los aspectos que deben ser evaluados? ¿Cuál es la información y decisiones que ya están disponibles?

En este paso se mostró a los jóvenes cómo identificar la idea de negocio, partiendo del análisis del entorno y las necesidades insatisfechas. Se hizo la identificación de ideas de negocio y los componentes básicos para poner en marcha un plan de trabajo, iniciándose con un proceso de priorización de ideas, de acuerdo con Rosero (2015); Durán et al. (2015); Córdoba e Izquierdo (2005).

\section{Proceso de priorización de ideas}

Para la evaluación de las ideas de negocio presentadas por los jóvenes, se hizo un taller para identificar los criterios y las características (Cuadro 1) que mostraron la pertinencia de la idea de negocio para la comunidad. A cada criterio se le asignó de manera participativa, una puntuación que iba de 1 a 5 , donde 1 significaba ninguna importancia de la idea de negocio para la comunidad y 5, es importante, entiende el concepto y tiene gran probabilidad de éxito, propuestas adaptadas de Rosero (2015); Durán et al. (2015).

\section{Análisis estadístico}

La información recolectada se sistematizó en el programa Excel, para importarla al programa SPAD-Win versión 5.6, posteriormente, se realizó un análisis de correspondencia múltiple (ACM), por tratarse de variables cualitativas, ya que son categorías exclusivas e independientes y se trabajó con el número de observaciones en cada categoría (Díaz et al., 2013).

\section{RESULTADOS Y DISCUSIÓN}

\section{Identificación de la capacidad de liderazgo de la mujer perteneciente a APROPAL}

De las 23 mujeres encuestadas, el 50\% están por encima de los 45 años, el 33\% está entre los 35 y 45 años y un $17 \%$ está entre los 25 y 35 años. Esta información permite ver que las actividades que se desarrollan en la asociación, están respaldadas y orientadas por diferentes puntos de vista generacionales, resaltándose un sentido de pertenencia, que se puede evidenciar en las mujeres de edad adulta que avivan constantemente la dinámica de la asociación.

De acuerdo con lo anterior, Perilla (2014) encontró que las mujeres más jóvenes apoyan las labores de 
Cuadro 1. Criterios que muestran la pertinencia de la idea de negocio para la comunidad.

Table 1. Criteria showing the relevance of the business idea for the community.

\begin{tabular}{ll}
\hline \multicolumn{1}{c}{ Criterio } & \multicolumn{1}{c}{ Características } \\
\hline Necesidad & $\begin{array}{l}\text { Medida como la capacidad para observar el entorno e identificar la carencia de algo } \\
\text { que se pueda satisfacer con un producto o servicio } \\
\text { Capacidad de relacionar la necesidad con el producto o servicio que se ofrece, y } \\
\text { Producto o servicio }\end{array}$ \\
creatividad para lograr el reconocimiento del mismo \\
Identificación de los posibles clientes del producto, con características como género, \\
edad, ubicación \\
Canales de distribución & $\begin{array}{l}\text { Descripción de la manera más adecuada para hacer llegar el producto hasta el cliente } \\
\text { Capacidad de la idea de negocio para generar más empleos para su comunidad }\end{array}$ \\
Disponibilidad de materia prima & $\begin{array}{l}\text { Capacidad de la idea de negocio para generar sostenibilidad por medio de la facilidad } \\
\text { de abastecimiento de la materia prima }\end{array}$ \\
\hline
\end{tabular}

Adaptado de Rosero (2015); Durán et al. (2015); Córdoba e Izquierdo (2005).

madres y abuelas en los quehaceres de la casa, como ha sido asignado socialmente, y lo que ellas consideran que deben realizar como mujeres y esposas, todo orientado especialmente al papel reproductivo. Aunque reconocen que los "tiempos han cambiado", y algunas participan en procesos productivos como el ser comerciantes (vendedoras en tiendas) y otros trabajos que les generan ingresos y "un poco más de independencia", expresan que el papel más importante es el de "ser madres y el cuidado de la familia".

No obstante, las más jóvenes y adultas jóvenes responden que ahora son agricultoras, cultivan y saben cómo se hace una huerta; han recibido capacitaciones para "ser más productivas", conformaron un hogar y actualmente se dedican al cuidado de los hijos y a las labores del hogar. Ahora son ellas quienes deciden -en su vida privada, en el hogar- y se sienten más importantes; consideran que hoy tienen los mismos derechos que los hombres (Perilla, 2014). En ese sentido, Bonilla (2010) afirma, que tanto las relaciones sociales como las de poder encuentran matices significativos si se considera el ciclo vital, entendido este como el conjunto de etapas o fases de la vida que si bien son delimitadas por la edad, se encuentran asociadas a procesos biológicos, organizados socialmente, que de manera similar al género, son mediatizados y re-significados socioculturalmente.

De otra parte, de las 23 mujeres encuestadas el 16\% tiene a su cargo entre 1 y 2 personas, el $62 \%$ entre 3 y 4 personas y el $22 \%$ entre 5 y 6 . De los datos anteriores se puede entender que un alto porcentaje de las mujeres asume una gran responsabilidad en la familia, considerándose así la columna vertebral del hogar, del trabajo campesino y de la subsistencia familiar cotidiana.

Estos datos concuerdan con los reportados por Sampedro (1996), Bonilla (2010) y Carrasco (2003), quie- nes afirman que dicha composición familiar -número de personas a cargo y edad- determina los compromisos de actividades a desarrollar y las responsabilidades sobre las mismas: “...el tamaño del hogar -dado por el número de miembros que lo integran- ejerce también una influencia considerable sobre la forma como las personas distribuyen su tiempo. En ese sentido, el hecho de tener niños pequeños, o personas mayores o enfermas que cuidar, limita naturalmente las posibilidades de disponer de tiempo para otras actividades, en particular, si estas labores tienen horarios o jornadas poco compatibles con las tareas de cuidado", circunstancia que ha restringido decididamente la movilidad de las mujeres.

En relación al grupo encuestado, se observó que el $66 \%$ de las mujeres de la asociación son casadas, el 13\% corresponde a mujeres solteras, el $8 \%$ de esta población está en unión libre y un 13\% de las mujeres encuestadas se hallan separadas (Figura 1). De los datos estadísticos obtenidos se comprobó que existe una marcada influencia y arraigo por lo tradicional, condición que trae como consecuencia el apego a la tierra, la transmisión de saberes y las costumbres ancestrales.

Con la investigación se pudo saber que el $18 \%$ de las asociadas toman la iniciativa en su familia, el $71 \%$ está a cargo del esposo, y el 11\% de los dos (Figura 2). Esta información concuerda con lo manifestado por Villarreal (2004), quien afirma que un elemento central en términos de pautas internas de relación que aún persisten en el mundo rural, es la predominancia de formas patriarcales, en donde la división sexual del trabajo marca la organización doméstica, generando patrones rígidos de control sobre las mujeres y los jóvenes.

Lo expuesto anteriormente, se corrobora por el mismo autor, quien expresa que la vida de las mujeres campesinas ha estado marcada por un trabajo duro, 
repetitivo, sin mayores alicientes y en condiciones de gran dificultad, que le significan muchas horas de agotadoras jornadas que incluyen la agricultura, la atención de los hijos, del marido y de otros parientes.

Respecto a los niveles de educación, en el grupo objeto de investigación se encontró que el $84 \%$ de las mujeres tiene primaria, un $8 \%$ básica secundaria, el $8 \%$ cuentan con educación media vocacional y el $8 \%$ restante no ha tenido acceso a la educación (Figura 3).
Es importante resaltar que el nivel educativo de las mujeres adscritas a la asociación en un porcentaje muy alto se circunscribe únicamente a la básica primaria, específicamente han cursado hasta tercer grado. La causa primordial de esta situación es la cultura. Al respecto, los padres consideraban que con tercer año escolar era más que suficiente para sus hijas, pues el objetivo se enmarcaba en saber leer y escribir, dando mayor prioridad a la ayuda en labores domésticas

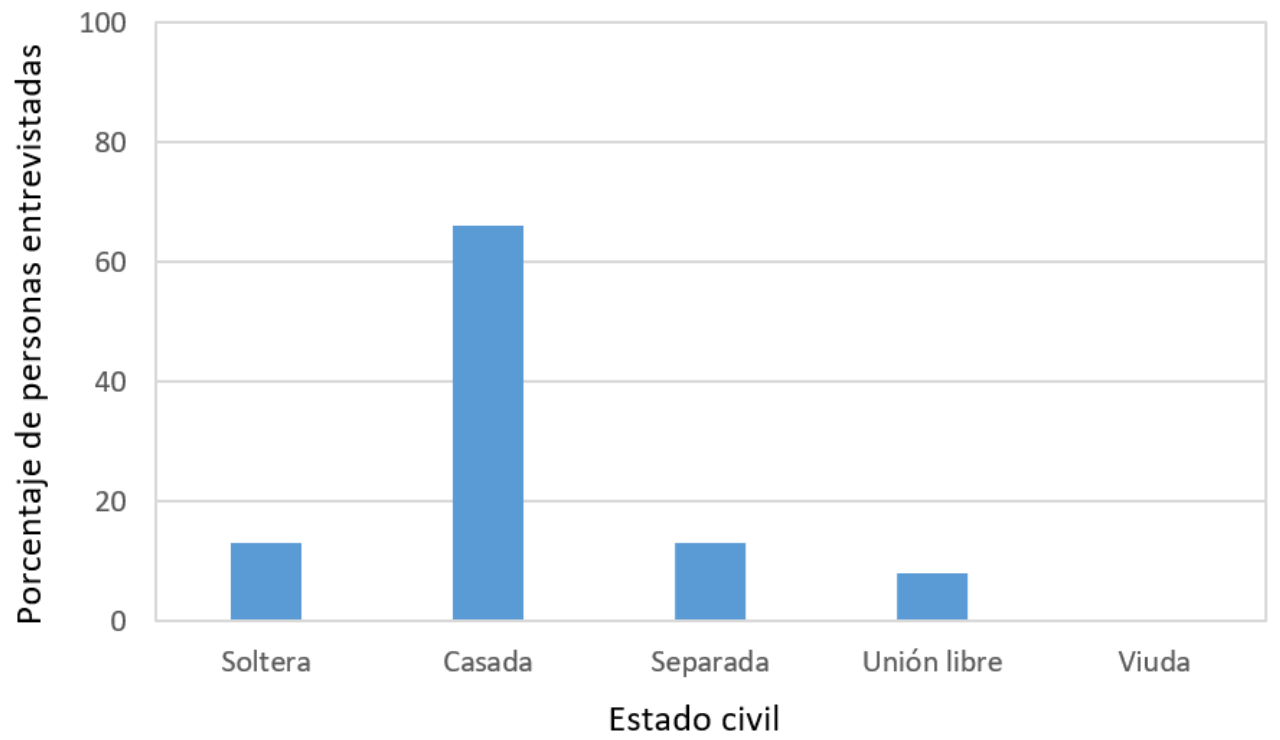

Figura 1. Conformación de la asociación, según su estado civil.

Figure 1. Associative conformation, according to marital status.

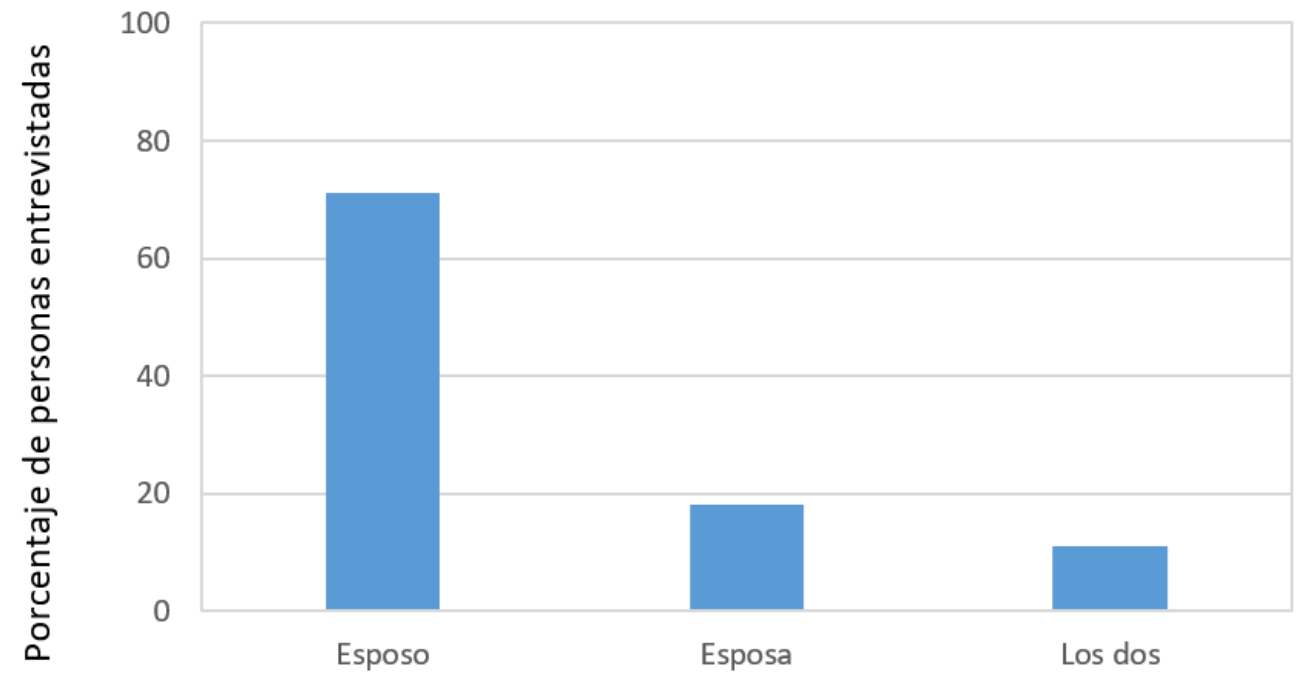

\section{Condición en el hogar}

Figura 2. Percepción del liderazgo al interior de la familia en la asociación.

Figure 2. Leadership perception within the family in the association. 


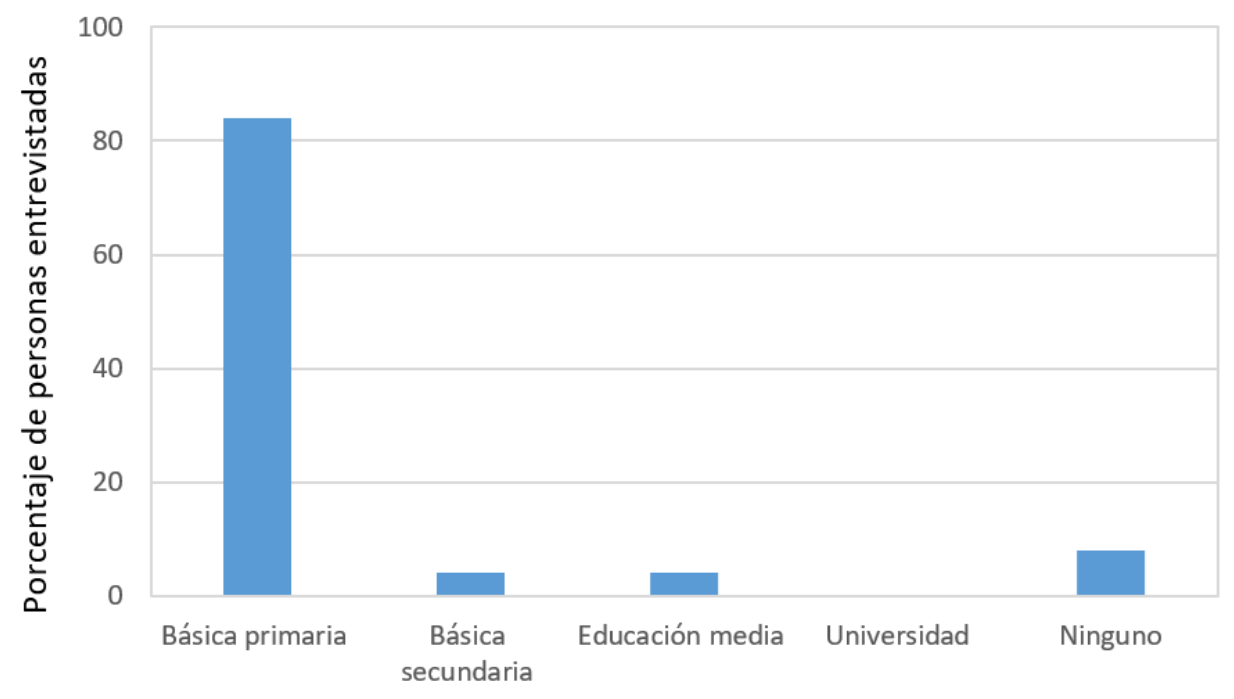

Niveles de educación

Figura 3. Nivel educativo de las mujeres pertenecientes a la asociación.

Figure 3. Educational level of women that are part of the association.

reafirmando el pensamiento colectivo masculino de "para cocinar no se necesita estudiar".

\section{Tipos de liderazgo en las mujeres pertenecientes a la asociación agropecuaria}

Fruto de la caracterización anteriormente detalla$\mathrm{da}$, se puede mencionar que si bien el componente participativo al interior de los hogares con respecto a la toma de decisiones es relativamente bajo, se han desarrollado mecanismos y estrategias de reflexión, análisis de la autoestima en lo personal y organizativo, que han incidido en las esferas familiares, comunitarias y regionales. Al respecto Burgos y Guerrero (2007) afirman que [Por primera vez en sus vidas es un tema que se piensa, se construye y se pone en la mesa para hablarlo, por ende, se crea la posibilidad de que] se empoderen luchando por un espacio de igualdad y de democracia.

El proceso de reflexión y análisis, que de forma continua viene desarrollando la asociación, contribuye para que desde el momento en que las mujeres participan en los talleres de organización comunitaria, propiciados por el proyecto, logren una apropiación del concepto expresándose de la siguiente manera: "Pues es que yo ya puedo ser líder, yo ya decido, es que yo ya voy para vicepresidenta. Es el querernos nosotras mismas, el ser líder es que los demás respeten nuestras decisiones y que nos queramos, que nos aceptemos como somos"2. De acuerdo con lo anterior, se encontraron diversos tipos de liderazgo que se discriminan a continuación.

\footnotetext{
${ }^{1}$ Dulce Carlosama, R. (15 de julio de 2016). Comunicación personal.

${ }^{2}$ Castillo Ordoñez, R. (09 de julio de 2016). Comunicación personal.
}

\section{Tipos de liderazgo en la mujer de la asociación}

Evaluada la totalidad de la información correspondiente a las 23 mujeres entrevistadas, se obtuvo que el 40,75\% de la variación de los datos (Figura 4), representa la presencia de un deseo conservacionista de la naturaleza, es decir, que dentro de la misma, se presentan tres características esenciales a saber: la mujer como productora y reproductora de la vida en relación con la naturaleza (componente 1), la perspectiva cultural de la organización (componente 2) y la visión de empoderamiento (componente 3).

El primer componente explicó que el 17,73\%, de la variabilidad de los datos obtenidos en relación a la totalidad de las mujeres entrevistadas, mostró que ellas se constituyen en productoras y reproductoras de vida en interrelación con la naturaleza, es por ello que, al hacer el análisis sobresalen las siguientes variables: practican la escucha activa (PEAC) $(\mathrm{V} 1=8,00)$, no toman decisiones sin haber estudiado todas las facetas del problema (NTDSHETFP) (V2 = 9,00), toman decisiones razonadas e involucran a sus colaboradores en el proyecto (TDRICEP) (V3 = 12,00), son buenas comunicadoras $(\mathrm{BC})(\mathrm{V} 4=17,00)$, generan confianza $(\mathrm{GC})$ (V5 = 11,00).

Por las variables analizadas, es posible afirmar que dentro de la asociación se encontraron lideres carismáticas, con características visionarias e inspiradoras, con ideales que apuntan al buen vivir de sus compañeras, familias y por ende de su comunidad, quienes según las entrevistas denotaron ser líderes con confianza en sí mismas y que por eso generan certidumbre en sus compañeras. 


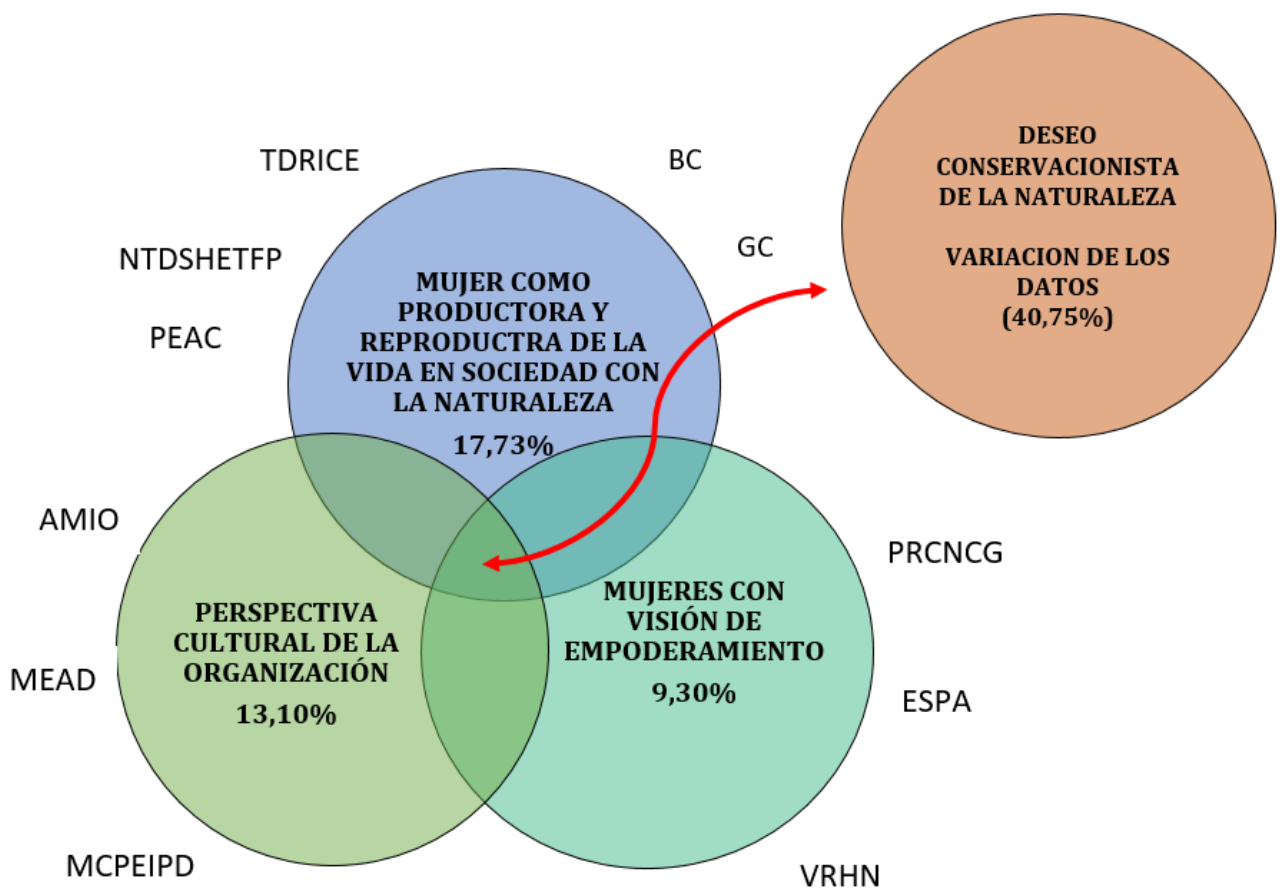

Figura 4. Integración de componentes para identificar los tipos de liderazgo en la asociación.

Figure 4. Integration of components to identify leadership types in the association.

Lo expuesto anteriormente concuerda, con lo planteado por Shiva (2014), quien afirma que este tipo de liderazgo, ubica a la mujer como conocedora de su propio derecho, a un conocimiento holístico y ecológico de los procesos de la naturaleza. En ese sentido Bass (1998), menciona que las mujeres con esa visión integral poseen convicciones sólidas, autoconfianza y presentan un fuerte anhelo de poder, además mantienen la confianza de sus seguidores, fortalecen el compromiso de los demás y muestran confianza en las habilidades de los asociados.

Lo anterior, lleva a afirmar que las mujeres de la asociación manejan valores basados en el bien común y la dignidad, siendo capaces de transformar a las personas con su discurso, todo esto para generar un equilibrio en su ambiente. Esta información corrobora lo expuesto por Puleo (2009), quien menciona que las mujeres no [son] las salvadoras del planeta ni las representantes privilegiadas de la naturaleza, pero pueden contribuir a un cambio sociocultural, que permita que las prácticas del cuidado de su entorno, que históricamente fueron sólo femeninas, se universalicen, es decir, que sean también propias de los hombres, y se extiendan al mundo natural no humano.

El segundo componente explicó el 13,10\% de la variabilidad total (23 mujeres). Este dato es significativo desde la agroecología, porque se relaciona con la perspectiva cultural de la organización y está representado por las siguientes variables: se avanza hacia una mayor igualdad de oportunidades (AMIO) (V6 $=7,70$ ), mayor espacio para la afirmación de la diferencia (MEAD) $(\mathrm{V} 7=8,90)$ y mayor capacidad de enriquecer el imaginario de participación democrática (MCPEIPD) (V8 = 10,00).

La información obtenida permite entender que las mujeres de la asociación tienen la capacidad de tomar decisiones, estimular la productividad, incrementar la identificación y satisfacción de los miembros de la organización, porque tienen destrezas a la hora de responder de manera eficiente a las demandas del ambiente.

El tercer componente reveló que el 9,30\% de la variabilidad de la información obtenida, se relaciona con la existencia de mujeres empoderadas de su quehacer en la cotidianidad. Las variables de mayor peso, es decir, las que contribuyeron a la conformación de este factor fueron: valoran el recurso humano y natural (VRHN) (V9 = 13,00), estimulan el sentido de la pertenencia a la asociación (ESPA) $(\mathrm{V} 10=7,90)$, promueven los resultados conjuntos y no crean competencia en el grupo (PRCNCG) $(\mathrm{V} 11=15,90)$.

Estas variables, demuestran la presencia de mujeres con características de líderes democráticos, que buscan la restructuración del pensamiento, de la cultura, de la visión de mundo, para así lograr alcanzar un ambiente sustentable, donde se pueda vivir y convivir con la naturaleza.

La anterior información concuerda con lo planteado por Villalva y Fierro (2017), quienes afirman que, este 
tipo de liderazgo permite crear mayor compromiso con la organización en la toma de decisiones, delegando autoridad y utilizando la retroalimentación como un medio para dirigir y corregir errores. Con base en lo expuesto, se encontró que los líderes democráticos imparten responsabilidades en la dirección del grupo, esto como principio de su direccionamiento, otorgándoles seguridad y respaldo a los miembros de la asociación, a su familia y a la comunidad. En conclusión, todas las mujeres de la asociación, trabajan con una visión compartida sobre la vida y un objetivo común, verbigracia, mejorar la calidad de vida y el buen vivir, así como visibilizar su accionar en la comunidad.

Fortalecimiento del emprendimiento juvenil, para la reedificación de sus objetivos y propósitos comunitarios

El resultado obtenido de las capacidades de emprendimiento en jóvenes se muestra en la Figura 5.

La Figura 5 muestra que existen cuatro habilidades emprendedoras, de las cuales tres son sobresalientes a saber: 1) La persistencia y capacidad para enfrentar problemas; 2) La determinación de metas; y 3) La autoconfianza. No obstante, se aprecia que los jóvenes tienen como debilidad un bajo aprovechamiento de nuevas oportunidades, ya sean de carácter académico o laboral.

En lo relacionado con el ítem denominado persistencia y capacidad para enfrentar los problemas, los miembros jóvenes de la asociación, presentan como destreza la cualidad de sortear las dificultades que se les han presentado a lo largo de los últimos años, como son la violencia y desplazamiento forzado entre otros. Al respecto Bayron Dulce ${ }^{3}$, hijo del señor representante legal de la asociación afirma que “... en las épocas de violencia, tuvimos mucho miedo porque las bombas llegaban hasta la escuela, por eso dejamos de ir a la escuela, ya no podíamos jugar ni salir al campo por temor a que nos lleven pal monte..."

Frente a lo anterior, los jóvenes de la comunidad y especialmente los vinculados a la asociación, manejaron las dificultades gracias a los fuertes lazos familiares y de arraigo a su tierra. Esto hizo que ellos desarrollaran capacidades adaptativas que les permitieron no ceder ante las adversidades económicas, sociales, ambientales y de violencia, creando en ellos condiciones y procesos de resiliencia.

Ahora bien, en lo atinente a la segunda habilidad que refiere acerca de la determinación de metas, es dable anotar que fruto de los procesos de resiliencia, los jóvenes de la localidad y especialmente los adscritos a la asociación, fijaron sus propios objetivos y metas en pro de construir un mejor futuro para ellos, sus ancestros y descendientes. Al respecto, el joven Alexander Viveros menciona que "... uno de los motivos para quedarme en mi casa y superar los problemas tuvo que ver con el amor a mi abuelita, para no dejarla sola lo cual permitió ponerme al frente de todas las tareas de la finca apersonándome del trabajo lo cual hizo que yo mirara un futuro en el campo para crecer..."

Con lo anterior se evidencia que los jóvenes de la asociación, además de ser persistentes son personas con una visión de futuro, máxime si se trabaja en equipo.

Respecto a la autoconfianza, es importante considerar que los jóvenes del municipio de Albán y especialmente los de la asociación, tienen un fuerte convencimiento para la realización con éxito de diferentes tareas, eligen el enfoque adecuado para la realización

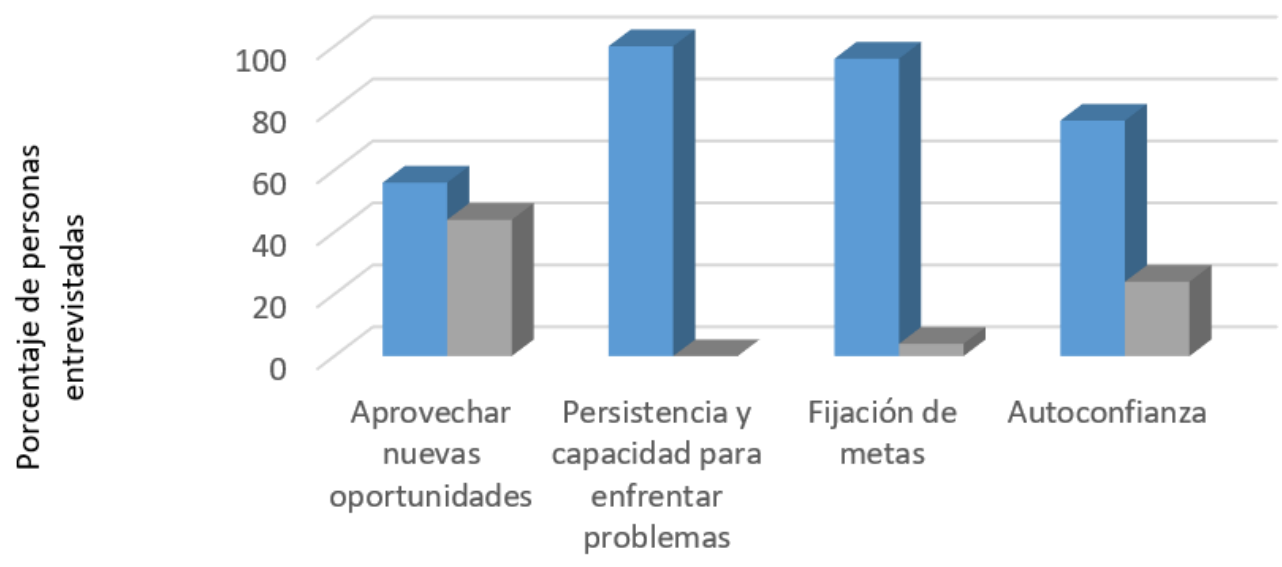

\% Participación fuerte — \% Participación débil

Figura 5. Habilidades emprendedoras en los jóvenes de la asociación.

Figure 5. Entrepreneurial skills of the young people that are part of the association.

\footnotetext{
${ }^{3}$ Dulce, B. (15 de agosto de 2016). Comunicación personal.
}

${ }^{4}$ Viveros, A. (12 de agosto de 2016). Comunicación personal. 
de un trabajo o para resolver las dificultades, además, muestran confianza en sus propias capacidades, todo esto, gracias al amor por su gente y su región. En ese sentido es importante dar a conocer un relato de autoconfianza expresado por Claudia Díaz (15 años) quien afirma que "...yo y mi hermano Jesús, nos anima mucho el ejemplo percibido de nuestros padres, esto nos dio confianza por lo que veíamos en su día a día, ellos madrugaban a la molienda y llegaban contentos y miramos que lo que hacían era bueno, porque no nos faltaba nada...". Todo lo anterior posibilita generar soluciones creativas, estimula la proactividad y la perseverancia, la confianza y la consecución de metas.

Finalmente, si se analiza la habilidad correspondiente al aprovechamiento de nuevas oportunidades, los jóvenes de la asociación manifestaron que si bien es cierto existen problemas en cuanto a oportunidades en el mercado laboral y educativo, ellos miran a la asociación como una alternativa viable para abrir espacios que les permita desarrollar sus capacidades de liderazgo, autogestión y emprendimiento. Al respecto Yuly Viveros ${ }^{6}$ dice "... a pesar de no contar con los espacios para desarrollar los procesos de formación, miramos que nosotros deseamos abrir camino para responder a las necesidades del municipio...".

Al respecto, el Instituto para el Desarrollo Rural de Sudamérica -IPDRS- (2017), considera que las condiciones estructurales de la ruralidad, tales como los altos índices de pobreza, las necesidades básicas insatisfechas y la ausencia de servicios públicos e infraestructura, acompañados de conflicto armado, han provocado que el campo cada vez esté más envejecido, pues los jóvenes han tenido que migrar a los centros poblados y ciudades en busca de mejores oportunidades de vida y realización personal, en cuanto a trabajo y educación.

Lo expuesto anteriormente concuerda con lo reportado por Jurado y Tobasura (2012), quienes afirman que "aunque el ámbito rural tiende a ser bien valorado como espacio de vida, las expectativas laborales y familiares y la identidad de los sujetos jóvenes parece construirse sobre el imaginario de la ciudad."

Por lo tanto, para enfrentar la problemática previamente descrita, es necesario desarrollar en la juventud Albanita, capacidades de liderazgo y competencias para la resolución de los problemas productivos, económicos, ambientales y sociales; para ello, se deberá promover y fortalecer la educación y de esta manera, aprovechar todo el potencial juvenil y con ello aterrizar las ideas que los jóvenes tienen para mejorar sus condiciones de vida y la de sus familias.

\footnotetext{
${ }^{5}$ Díaz, C. (24 de agosto de 2016). Comunicación personal.

${ }^{6}$ Viveros, Y. (24 de agosto de 2016). Comunicación personal.
}

\section{Identificación y desarrollo de ideas de negocio de los jóvenes Albanitas}

Los jóvenes presentaron sus ideas acordes a negocios que no existen en su comunidad (Cuadro 2 y 3 ).

La idea de negocio que tiene mayor puntaje en la matriz de priorización en los jóvenes es la número I, denominada producción de abonos orgánicos y caldos microbianos de nombre "El agricultor albanita". Esta alternativa, tuvo una gran acogida porque los jóvenes han detectado en la asociación, un espacio adecuado para desarrollar sus ideas y empoderarse del quehacer de la misma. En ese sentido, la información anterior concuerda con lo manifestado por Zapata (2000), quien afirma que el joven rural tiene una percepción de las posibles áreas de trabajo, por lo que se puede afirmar que el campo laboral de ellos se estructura en torno a lo agrícola.

Cuadro 2. Ideas de negocio de los jóvenes pertenecientes a la asociación.

Table 2. Business ideas of the young people that are part of the association.

\begin{tabular}{|c|c|c|}
\hline No & Ideas de negocio & Nombre \\
\hline I & Producción de abonos orgánicos & $\begin{array}{l}\text { El agricultor } \\
\text { albanita }\end{array}$ \\
\hline II & Producción de panela pulverizada & Solo Albanita \\
\hline III & Comidas rápidas & Natuburger \\
\hline IV & Gimnasio & Gymuniversal \\
\hline V & Punto de venta de cosmetología & $\begin{array}{l}\text { Cosmetología } \\
\text { El triunfo }\end{array}$ \\
\hline
\end{tabular}

Cuadro 3. Matriz de priorización de ideas de negocio de los jóvenes pertenecientes a la asociación.

Table 3. Prioritization matrix of business ideas from young people that are part of the association.

\begin{tabular}{lccccc}
\hline \multirow{2}{*}{\multicolumn{1}{c}{ Atributos }} & \multicolumn{5}{c}{ Ideas de negocio } \\
\cline { 2 - 7 } & I & II & III & IV & V \\
\hline Necesidad & 5 & 4 & 4 & 2 & 4 \\
Producto o servicio & 4 & 4 & 3 & 2 & 4 \\
Descripción del cliente & 4 & 3 & 2 & 3 & 2 \\
Utilización del canal de distribución & 3 & 3 & 3 & 4 & 1 \\
Generación de empleo & 3 & 2 & 2 & 2 & 2 \\
Disponibilidad de materia prima & 4 & 2 & 3 & 2 & 2 \\
\hline \multicolumn{1}{c}{ TOTAL } & $\mathbf{2 3}$ & $\mathbf{1 8}$ & $\mathbf{1 7}$ & $\mathbf{1 5}$ & $\mathbf{1 5}$ \\
\hline
\end{tabular}




\section{CONCLUSIONES}

Se evidenció en las mujeres de la Asociación Agropecuaria APROPAL, una visión de empoderamiento y una perspectiva cultural de la organización, que se apoya en su calidad de productoras y reproductoras de vida, e induce en la asociación el deseo conservacionista de la naturaleza.

El fortalecimiento del liderazgo de las mujeres y jóvenes de APROPAL, es un elemento estratégico para mejorar la gestión asociativa en el sector agroproductivo regional y constituye un aspecto complementario al fortalecer el tejido empresarial de la región.

La participación de las mujeres y jóvenes que hacen parte de la APROPAL, los convierte en importantes y sólidos actores de la vida local rural y abre nuevas vías de participación social y de influencia pública, al canalizar gran diversidad de potencialidades e intereses en la región.

\section{REFERENCIAS}

Acuña, M., 2013. El fenómeno del desplazamiento forzado en Colombia: Departamento del Magdalena uno de los afectados.Erg@omnes 5(1), 17-32.

Alpargatero, L., 2011. La política pública de desplazamiento forzado en Colombia: una visión del pensamiento complejo. Editorial Universidad de Rosario, Escuela de Administración, Bogotá, Colombia.

Alto Comisionado de Naciones Unidas para los Refugiados (ACNUR), 2010. Tendencias globales 2009: Refugiados, solicitantes de asilo, retornados, desplazados internos y personas apátridas. División de Administración y Apoyo a Programas, ACNUR, Ginebra, Suiza.

Bass, B., 1998. Transformational leadership: industry, military and educational impact. Lawrence Erlbaum Associates, Inc., New York.

Benítez, G., 2010. La mujer rural: derechos, desafíos y perspectivas. Memorias del conversatorio internacional La mujer rural: derechos, desafíos y perspectivas. Centro de Investigación y Educación Popular (Cinep), 7-9 de Julio de 2010, Bogotá, Colombia, pp. 19-105. http:// datoscolombianuncamas.org/images/abook_file/mujer_rural.pdf

Bonilla, A., 2010. Trabajo doméstico y mujer rural: ...ésta vida mía. Tesis de Grado Maestra en Estudios de Género, Área Mujer y Desarrollo, Universidad Nacional de Colombia, Bogotá. 143 p.

Burgos, M., Guerrero, S., 2007. Caracterización de la capacidad de liderazgo de la mujer perteneciente al grupo asociativo AMTTA - Asociación de Mujeres Trabajadoras de Tangua - en el departamento de Nariño. Tesis de grado de Especialista en Gerencia Social, Universidad de Nariño, Colombia. 108 p.

Carrasco, C., 2003. Los tiempos de trabajo: entre la casa y el mercado: nuevas aproximaciones de análisis de resultados. Universidad de Barcelona, Barcelona, España.

Castro, P., Escobar, P., 2015. Análisis del desarrollo de la capacidad emprendedora de los estudiantes del programa de Química Industrial de la Universidad Tecnológica de Pereira a lo largo de su formación académica. Tesis de grado de Tecnología Industrial, Universidad Tecnológica de Pereira, Colombia. 137 p.

Cediel, N.M., Donoso, N., Hernández, J., López, M.C., Herrera, P., Moreno, C., 2017. Empoderamiento de las mujeres rurales como gestoras de los objetivos de desarrollo sostenible en el posconflicto colombiano. Equidad y Desarrollo 28, 61-84. http://dx.doi.org/10.19052/ed.4077

Córdoba, M., Izquierdo, S., 2005. Caracterización socio-familiar de los niños, niñas y jóvenes del corregimiento el Manzano, municipio de Taminango en el periodo enerojulio 2005. Tesis de grado de Sociólogo. Universidad de Nariño, Colombia. 123 p.

Díaz, M., González, A.L., Henao, A., Díaz, M.E., 2013. Introducción al análisis estadístico multivariado aplicado: experiencia y casos en el Caribe colombiano. Editorial Universidad del Norte, Área Metropolitana de Barranquilla, Colombia.

Durán, S., Parra, M., Márceles, V., 2015. Potenciación de habilidades para el desarrollo de emprendedores exitosos en el contexto universitario. Opción 31(77) 200-215.

Farah, M.A., Pérez, E., 2003. Mujeres rurales y nueva ruralidad en Colombia. Cuadernos de Desarrollo Rural 51, 137-160.

Geilfus, F., 2002. 80 herramientas para el desarrollo participativo: diagnóstico, planificación, monitoreo y evaluación. Instituto Interamericano de Cooperación para la Agricultura (IICA), Costa Rica.

Gómez, Y., 2016. Un gobierno de puertas abiertas al progreso. Plan de Desarrollo Municipal 2016-2019, San José de Albán, Nariño.

González, R., Trejos, L., Vargas, A., Zuluaga, J., Guzmán, C., Molinares, I., Navarro, L., Zubiría, B., Tous, J., Monsalvo, E., Cervantes, G., San Juan, H., Acosta, J., Coscione, M., Montero, P., 2016. ¿Fin del conflicto armado en Colombia?: escenarios de postacuerdo. Universidad del Norte, Grupo Editorial Ibañez, Área Metropolitana de Barranquilla, Colombia.

Güechá, E., Sastre, S., y Forero, K., 2017. El rol de los jóvenes en el fomento del desarrollo rural en Colombia. Tesis de Grado de Profesional de Finanzas y Comercio Internacional, Universidad de la Salle, Facultad de Ciencias Económicas y Sociales, Bogotá. 65 p.

Instituto para el Desarrollo Rural de Sudamérica (IPDRS), 2017. La juventud rural en Colombia y sus condiciones de acceso y permanencia en la tierra. http://comosoc.org/La-juventud-rural-en-Colombia-y (acceso, 30.07.2017).

Jurado, C., Tobasura, I., 2012. Dilema de la juventud en territorios rurales de Colombia: ¿campo o ciudad? Revista Latinoamericana de Ciencias Sociales, Niñez y Juventud 10(1), 63-77.

Montenegro, M., 2012. Caracterización de la diversidad fitogenética de los huertos caseros en el corregimiento el ingenio, municipio de Ancuya, departamento de Nariño. Tesis de Maestría en Ciencias Agrarias, Universidad de Nariño, Colombia. 150 p.

Perilla, L., 2014. Los roles de las mujeres rurales en el departamento de Nariño, Colombia: tendencias y cambios. Trabajo Social 16(16), 187-204. 
Puleo, A., 2009. Ecofeminismo: la perspectiva de género en la conciencia ecologista, en: Riechmann, J., Taibo, C., Fernández, R., Puleo, A., Alguacil, J., Hernández, A., Rois, C., Zamarra, C., López, D., Llistar, D., Romano, D., Pérez, E., Gómez-Baggethun, E., Aneiros, E., Cembranos, F., Castejón, F., Tarafa, G., Barcena, I., Barajas, J., Barcia, J.V., del Olmo, J.C., González, L., García-Amado, L., Gimena, M., González, M., Pascual, M., Soler, M., Segura, P., Lago, R., Oberhuber, T., Herrero, Y. (Eds.), Claves del ecologismo social. Ecologistas en Acción, Madrid, 169-172.

Rosero, D., 2015. Fortalecimiento de capacidades empresariales a jóvenes de la institución educativa ecológica La Cocha, municipio de Arboleda, Departamento de Nariño. Tesis Grado de Profesional en Comercio Internacional y Mercadeo, Universidad de Nariño. 82 p.

Sampedro, R., 1996. Género y ruralidad: las mujeres ante el reto de la desagrarización. Instituto de la Mujer, Ministerio de Trabajo y Asuntos Sociales, España.

Shiva, V., 2014. Abrazar la vida: mujer, ecología y desarrollo.
Editorial Horas y Horas, Madrid, España.

Somarriba, E., 2009. Planificación agroforestal de fincas. Centro Agronómico Tropical de Investigación y Enseñanza (CATIE), Turrialba, Costa Rica, 102 p. http://simas.org. ni/media/PAF_Libro_Impreso.pdf

Villalva, M., Fierro, I., 2017. El liderazgo democrático: una aproximación conceptual. INNOVA Research Journal 2(4), 155-162.

Villarreal, N., 2004. Sectores campesinos mujeres rurales y estado en Colombia. Tesis de Doctorado en Sociología, Universidad Autónoma de Barcelona, Facultad de Ciencias Políticas y Sociología, España. 524 p.

Zapata, S., 2000. Al encuentro del joven rural. Instituto Interamericano de Cooperación para la Agricultura (IICA), Agencia de Cooperación del IICA en Chile, Santiago, Chile.

Zuluaga-Sánchez, G., Arango-Vargas, C., 2013. Mujeres campesinas: resistencia, organización y agroecología en medio del conflicto armado. Cuadernos de Desarrollo Rural 10 (72), 159-180. 
